\title{
DIFFERENTIAL SUBORDINATIONS FOR CLASSES OF MEROMORPHIC $p$-VALENT FUNCTIONS DEFINED BY MULTIPLIER TRANSFORMATIONS
}

\author{
R. M. EL-ASHWAH, M. K. AOUF and T. BULBOACĂ ${ }^{凶}$
}

(Received 28 September 2009)

\begin{abstract}
We investigate several inclusion relationships and other interesting properties of certain subclasses of $p$-valent meromorphic functions, which are defined by using a certain linear operator, involving the generalized multiplier transformations.
\end{abstract}

2010 Mathematics subject classification: primary 30C45; secondary 30C80.

Keywords and phrases: multiplier transformations, meromorphic functions, differential subordination.

\section{Introduction}

For $n>-p$, let $\sum_{p, n}$ denote the class of meromorphic functions of the form

$$
f(z)=z^{-p}+\sum_{k=n}^{\infty} a_{k} z^{k}, \quad p \in \mathbb{N}=\{1,2,3, \ldots\},
$$

which are analytic and $p$-valent in the punctured unit disc $\dot{U}=U \backslash\{0\}$, where $U=$ $\{z \in \mathbb{C}:|z|<1\}$. For convenience, we write $\sum_{p} \equiv \sum_{p,-p+1}$.

If $f$ and $g$ are two analytic functions in $U$, we say that $f$ is subordinate to $g$, written symbolically as $f(z) \prec g(z)$, if there exists a Schwarz function $w$, which (by definition) is analytic in $U$ with $w(0)=0$, and $|w(z)|<1, z \in U$, such that $f(z)=g(w(z))$ for all $z \in U$.

It is well known that, if $f(z) \prec g(z)$, then $f(0)=g(0)$ and $f(U) \subset g(U)$. Further, if the function $g$ is univalent in $U$, then we have the following equivalence (see [9]; see also [10, p. 4]):

$$
f(z) \prec g(z) \Leftrightarrow f(0)=g(0) \quad \text { and } \quad f(U) \prec g(U) .
$$

For the functions $f_{j} \in \sum_{p, n}, j=1,2$, given by

$$
f_{j}(z)=z^{-p}+\sum_{k=n}^{\infty} a_{k, j} z^{k}
$$

(C) 2011 Australian Mathematical Publishing Association Inc. 0004-9727/2011 \$16.00 
we define the Hadamard (or convolution) product of $f_{1}$ and $f_{2}$ by

$$
\left(f_{1} * f_{2}\right)(z)=z^{-p}+\sum_{k=n}^{\infty} a_{k, 1} a_{k, 2} z^{n}
$$

Define the linear operator $I_{p}^{m}(n ; \lambda, l): \sum_{p, n} \rightarrow \sum_{p, n}$, where $\lambda \geq 0, l>0$, and $m \in \mathbb{N}_{0}=\mathbb{N} \cup\{0\}$, by

$$
I_{p}^{m}(n ; \lambda, l) f(z)=z^{-p}+\sum_{k=n}^{\infty}\left[\frac{\lambda(k+p)+l}{l}\right]^{m} a_{k} z^{k} .
$$

Then, we can write (1.1) as

$$
I_{p}^{m}(n ; \lambda, l) f(z)=\left(\Phi_{n ; \lambda, l}^{p, m} * f\right)(z),
$$

where

$$
\Phi_{n ; \lambda, l}^{p, m}(z)=z^{-p}+\sum_{k=n}^{\infty}\left[\frac{\lambda(k+p)+l}{l}\right]^{m} z^{k} .
$$

Using definition (1.1), it is easy to verify that the next formula holds for $\lambda>0$ :

$$
\lambda z\left(I_{p}^{m}(n ; \lambda, l) f(z)\right)^{\prime}=l I_{p}^{m+1}(n ; \lambda, l) f(z)-(\lambda p+l) I_{p}^{m}(n ; \lambda, l) f(z) .
$$

REMARK 1.1. (1) We note that $I_{p}^{0}(n ; \lambda, l) f=f$ and

$$
I_{p}^{1}(n ; 1,1) f(z)=\frac{\left(z^{p+1} f(z)\right)^{\prime}}{z^{p}}=(p+1) f(z)+z f^{\prime}(z) .
$$

(2) For some special values of the parameters $\lambda, l, m$ and $p$, we obtain the following operators studied by various authors:

(i) $I_{p}^{m}(n ; 1, l)=I_{p}^{m}(n, l)$ (see Cho et al. [2]);

(ii) $I_{p}^{m}(n ; 1,1)=D_{n, p}^{m}$ (see Aouf and Hossen [1], and Liu and Srivastava [6]);

(iii) $I_{1}^{m}(0 ; 1, l)=D_{l}^{m}$ (see Cho et al. $\left.[3,4]\right)$;

(iv) $I_{1}^{m}(0 ; 1,1)=I^{m}$ (see Uralegaddi and Somanatha [18]).

Using differential subordinations as well as the linear operator $I_{p}^{m}(n ; \lambda, l)$, we will introduce a subclass of $\sum_{p, n}$, as follows.

Definition 1.2. (1) For the fixed parameters $A$ and $B$, with $-1 \leq B<A \leq 1$, we say that a function $f \in \sum_{p, n}$ is in the class $\sum_{p, n}^{m}(\lambda, l ; A, B)$, if it satisfies the subordination condition

$$
-\frac{z^{p+1}\left(I_{p}^{m}(n ; \lambda, l) f(z)\right)^{\prime}}{p} \prec \frac{1+A z}{1+B z}, \quad l, \lambda>0, m \in \mathbb{N}_{0}, n>-p .
$$

(2) For convenience, we write

$$
\sum_{p, n}^{m}(\lambda, l ; \alpha) \equiv \sum_{p, n}^{m}\left(\lambda, l ; 1-\frac{2 \alpha}{p},-1\right), \quad 0 \leq \alpha<p,
$$


that is, $\sum_{p, n}^{m}(\lambda, l ; \alpha)$ denotes the class of functions $f \in \sum_{p, n}$ satisfying

$$
\operatorname{Re}\left\{-z^{p+1}\left(I_{p}^{m}(n ; \lambda, l) f(z)\right)^{\prime}\right\}>\alpha, \quad z \in U .
$$

REMARK 1.3. We have the next special cases of $\sum_{p, n}^{m}(\lambda, l ; A, B)$, studied previously by different authors:

(i) $\quad \sum_{p, 0}^{m}(1,1 ; A, B)=R_{m, p}(A, B)$ (see Liu and Srivastava [6]);

(ii) $\quad \sum_{p, n}^{m}(1,1 ; A, B)=\sum_{p, n}^{m}(A, B)$ (see Srivastava and Patel [16]);

(iii) $\sum_{p, 0}^{0}(1,1 ; A, B)=H(p ; A, B)($ see Mogra $[11,12])$;

(iv) $\sum_{p, n}^{m}(1, l ; A, B)=\sum_{p, n}^{m, l}(A, B)$, where $\sum_{p, n}^{m, l}(A, B)$ is the class of functions $f \in \sum_{p, n}$, satisfying

$$
-\frac{z^{p+1}\left(I_{p}^{m}(n, l) f(z)\right)^{\prime}}{p} \prec \frac{1+A z}{1+B z}, \quad l>0, m \in \mathbb{N}_{0}, n>-p,
$$

and $I_{p}^{m}(n, l) \equiv I_{p}^{m}(n ; 1, l)$.

In the present paper we obtain several inclusion relationships for the function class $\sum_{p, n}^{m}(\lambda, l ; A, B)$, and we investigate various other properties of functions belonging to the class $\sum_{p, n}^{m}(\lambda, l ; A, B)$. Relevant connections of the results presented in this paper with those obtained in earlier works are also pointed out.

\section{Preliminaries}

To establish our main results, we will need the following lemmas and definition.

LEMMA 2.1 [5]. Let the function $h$ be convex (univalent) in $U$, with $h(0)=1$. Suppose also that the function $\varphi$ given by

$$
\varphi(z)=1+c_{p+n} z^{p+n}+c_{p+n+1} z^{p+n+1}+\cdots
$$

is analytic in $U$. Then

$$
\varphi(z)+\frac{z \varphi^{\prime}(z)}{\delta} \prec h(z), \quad \operatorname{Re} \delta \geq 0, \delta \neq 0,
$$

implies that

$$
\varphi(z) \prec \psi(z)=\frac{\delta}{p+n} z^{-\delta /(p+n)} \int_{0}^{z} t^{\delta /(p+n)-1} h(t) d t \prec h(z),
$$

and $\psi$ is the best dominant of (2.2).

DEFINITION 2.2. We denote by $\mathcal{P}(\gamma)$ the class of functions $\varphi$ given by

$$
\varphi(z)=1+b_{1} z+b_{2} z^{2}+\cdots,
$$

which are analytic in $U$ and satisfy the inequality

$$
\operatorname{Re} \varphi(z)>\gamma, \quad z \in U(0 \leq \gamma<1) .
$$


LEMMA 2.3 [14]. Let the function $\varphi$ given by (2.3) be in the class $\mathcal{P}(\gamma)$. Then

$$
\operatorname{Re} \varphi(z) \geq 2 \gamma-1+\frac{2(1-\gamma)}{1+|z|}, \quad z \in U(0 \leq \gamma<1) .
$$

LEMMA 2.4 [17]. For $0 \leq \gamma_{1}<\gamma_{2}<1$, the inclusion

$$
\mathcal{P}\left(\gamma_{1}\right) * \mathcal{P}\left(\gamma_{2}\right) \subset \mathcal{P}\left(\gamma_{3}\right) \quad \text { where } \gamma_{3}=1-2\left(1-\gamma_{1}\right)\left(1-\gamma_{2}\right) \text {, }
$$

holds and the result is the best possible. The symbol '*' stands for the previous mentioned Hadamard product of the power series.

LEMMA 2.5 [15]. Let $\Phi$ be an analytic function in $U$, with $\Phi(0)=1$ and $\operatorname{Re} \Phi(z)>$ $1 / 2, z \in U$. Then, for any function $F$ analytic in $U$, the set $(\Phi * F)(U)$ is contained in the convex hull of $F(U)$, that is, $(\Phi * F)(U) \subset \operatorname{co} F(U)$.

LEMMA 2.6 [19]. For all real or complex numbers $\alpha_{1}, \alpha_{2}, \beta_{1}$, where $\beta_{1} \notin \mathbb{Z}_{0}^{-}=$ $\{0,-1,-2, \ldots\}$,

$$
\begin{aligned}
& \int_{0}^{1} t^{\alpha_{2}-1}(1-t)^{\beta_{1}-\alpha_{2}-1}(1-z t)^{-\alpha_{1}} d t \\
& =\frac{\Gamma\left(\alpha_{2}\right) \Gamma\left(\beta_{1}-\alpha_{2}\right)}{\Gamma\left(\beta_{1}\right)}{ }_{2} F_{1}\left(\alpha_{1}, \alpha_{2}, \beta_{1} ; z\right) \text { for } \operatorname{Re} \beta_{1}>\operatorname{Re} \alpha_{2}>0 \\
& \quad{ }_{2} F_{1}\left(\alpha_{1}, \alpha_{2}, \beta_{1} ; z\right)={ }_{2} F_{1}\left(\alpha_{2}, \alpha_{1}, \beta_{1} ; z\right) \\
& { }_{2} F_{1}\left(\alpha_{1}, \alpha_{2}, \beta_{1} ; z\right)=(1-z)^{-\alpha_{1}}{ }_{2} F_{1}\left(\alpha_{1}, \beta_{1}-\alpha_{2}, \beta_{1} ; \frac{z}{z-1}\right)
\end{aligned}
$$

and

$$
{ }_{2} F_{1}\left(\alpha_{1}, \alpha_{2}, \frac{\alpha_{1}+\alpha_{2}+1}{2} ; \frac{1}{2}\right)=\frac{\sqrt{\pi} \Gamma\left(\frac{\alpha_{1}+\alpha_{2}+1}{2}\right)}{\Gamma\left(\frac{\alpha_{1}+1}{2}\right) \Gamma\left(\frac{\alpha_{2}+1}{2}\right)},
$$

where ${ }_{2} F_{1}$ represents the Gauss hypergeometric function.

\section{Subordination theorems and the associated functional inequalities}

Unless otherwise mentioned, we shall assume throughout the paper that $n$ is an integer with $n>-p$, that $-1 \leq B<A \leq 1, \lambda, l>0, m \in \mathbb{N}_{0}, \beta>0$, and $p \in \mathbb{N}$.

THEOREM 3.1. If the function $f \in \sum_{p, n}$ satisfies the subordination condition

$$
-\frac{(1-\beta) z^{p+1}\left(I_{p}^{m}(n ; \lambda, l) f(z)\right)^{\prime}+\beta z^{p+1}\left(I_{p}^{m+1}(n ; \lambda, l) f(z)\right)^{\prime}}{p} \prec \frac{1+A z}{1+B z},
$$

then

$$
-\frac{z^{p+1}\left(I_{p}^{m}(n ; \lambda, l) f(z)\right)^{\prime}}{p} \prec Q(z) \prec \frac{1+A z}{1+B z},
$$


where the function $Q$ is given by

$$
Q(z)= \begin{cases}\frac{A}{B}+\left(1-\frac{A}{B}\right)(1+B z)_{2}^{-1} F_{1}\left(1,1, \frac{l}{\lambda \beta(p+n)}+1 ; \frac{B z}{1+B z}\right), & B \neq 0, \\ 1+\frac{l}{\lambda \beta(p+n)+l} A z, & B=0,\end{cases}
$$

and it is the best dominant of (3.1).

Furthermore, for all $k \in \mathbb{N}$, we have

$$
\operatorname{Re}\left[-\frac{z^{p+1}\left(I_{p}^{m}(n ; \lambda, l) f(z)\right)^{\prime}}{p}\right]^{1 / k}>\rho^{1 / k}, \quad z \in U,
$$

where $\rho=Q(-1)$, and the inequality (3.2) is the best possible.

PROOF. If we consider the function $\varphi$ defined by

$$
\varphi(z)=-\frac{z^{p+1}\left(I_{p}^{m}(n ; \lambda, l) f(z)\right)^{\prime}}{p},
$$

then $\varphi$ has the form (2.1) and is analytic in $U$. Applying the identity (1.2) in (3.3), and differentiating the resulting equation with respect to $z$, we get

$$
\begin{gathered}
-\frac{(1-\beta) z^{p+1}\left(I_{p}^{m}(n ; \lambda, l) f(z)\right)^{\prime}+\beta z^{p+1}\left(I_{p}^{m+1}(n ; \lambda, l) f(z)\right)^{\prime}}{p} \\
=\varphi(z)+\frac{\beta \lambda}{l} z \varphi^{\prime}(z) \prec \frac{1+A z}{1+B z} .
\end{gathered}
$$

Now by using Lemma 2.1 for $\gamma=l /(\lambda \beta)$, we deduce that

$$
\begin{aligned}
& -\frac{z^{p+1}\left(I_{p}^{m}(n ; \lambda, l) f(z)\right)^{\prime}}{p} \prec Q(z) \\
& \quad=\frac{l}{\lambda \beta(p+n)} z^{-l / \lambda \beta(p+n)} \int_{0}^{z} t^{(l / \lambda \beta(p+n))-1} \frac{1+A t}{1+B t} d t \\
& = \begin{cases}\frac{A}{B}+\left(1-\frac{A}{B}\right)(1+B z)^{-1}{ }_{2} F_{1}\left(1,1, \frac{l}{\lambda \beta(p+n)}+1 ; \frac{B z}{1+B z}\right), & B \neq 0, \\
1+\frac{l}{\lambda \beta(p+n)+l} A z, & B=0,\end{cases}
\end{aligned}
$$

where we made a changes of variables, followed by the use of the identities (2.4), (2.5), and (2.6) (with $b=1$ and $c=a+1$ ). Hence, assertion (3.1) is proved.

In order to prove assertion (3.2), it is sufficient to show that

$$
\inf \{\operatorname{Re} Q(z):|z|<1\}=Q(-1) .
$$


Indeed, for $|z| \leq r<1$,

$$
\operatorname{Re} \frac{1+A z}{1+B z} \geq \frac{1-A r}{1-B r}, \quad|z| \leq r<1 .
$$

Setting

$$
G(s, z)=\frac{1+A s z}{1+B s z}
$$

and

$$
d \nu(s)=\frac{l}{\lambda \beta(p+n)} s^{l / \lambda \beta(p+n)} d s, \quad 0 \leq s \leq 1,
$$

which is a positive measure on $[0,1]$, we get

$$
Q(z)=\int_{0}^{1} G(s, z) d v(s),
$$

so that

$$
\operatorname{Re} Q(z) \geq \int_{0}^{1} \frac{1-A s r}{1-B s r} d v(s)=Q(-r), \quad|z| \leq r<1 .
$$

Letting $r \rightarrow 1^{-}$in the above inequality, and using the elementary inequality

$$
\operatorname{Re} w^{1 / k} \geq(\operatorname{Re} w)^{1 / k}, \quad \operatorname{Re} w>0, k \in \mathbb{N},
$$

we obtain (3.2). Finally, inequality (3.2) is the best possible, as the function $Q$ is the best dominant of (3.1).

Remark 3.2. Putting $\lambda=l=1$ in Theorem 3.1, we obtain the result of Srivastava and Patel [16, Theorem 1].

For $\lambda=l=1, n=0$, and $\beta=1$, Theorem 3.1 yields the following result, which improves the corresponding one of Liu and Srivastava [7, Theorem 1].

COROLlary 3.3. The inclusions

$$
R_{m+1, p}(A, B) \subset R_{m, p}(A, B) \subset R_{m, p}(1-2 \rho,-1)
$$

hold, where

$$
\rho= \begin{cases}\frac{A}{B}+\left(1-\frac{A}{B}\right)(1-B)^{-1} F_{1}\left(1,1, \frac{1}{p}+1 ; \frac{B}{B-1}\right), & B \neq 0, \\ 1-\frac{A}{p+1}, & B=0,\end{cases}
$$

and the result is the best possible.

Putting $A=1-2 \alpha / p, \quad B=-1, \quad \beta=\lambda=l=1, \quad m=0$ and $n=-p+2$ in Theorem 3.1, and using (2.7), we get the following result. 
COROLlary 3.4. If the function $f \in \sum_{p,-p+2}$ satisfies the inequality

$$
\operatorname{Re}\left\{-z^{p+1}\left[(p+2) f^{\prime}(z)+z f^{\prime \prime}(z)\right]\right\}>\alpha, \quad z \in U(0 \leq \alpha<p),
$$

then

$$
\operatorname{Re}\left[-z^{p+1} f^{\prime}(z)\right]>\alpha+(p-\alpha)\left(\frac{\pi}{2}-1\right), \quad z \in U,
$$

and the result is the best possible.

REMARK 3.5. Taking $\alpha=-p(\pi-2) /(4-\pi)$ in the above corollary, we obtain that if the function $f \in \sum_{p,-p+2}$ satisfies

$$
\operatorname{Re}\left\{-z^{p+1}\left[(p+2) f^{\prime}(z)+z f^{\prime \prime}(z)\right]\right\}>-\frac{p(\pi-2)}{4-\pi}, \quad z \in U,
$$

then $\operatorname{Re}\left[-z^{p+1} f^{\prime}(z)\right]>0, z \in U$ (see Pap [13]).

THEOREM 3.6. If the function $f \in \sum_{p, n}^{m}(\lambda, l ; \alpha), 0 \leq \alpha<p$, then

$$
\operatorname{Re}\left\{-z^{p+1}\left[(1-\beta)\left(I_{p}^{m}(n ; \lambda, l) f(z)\right)^{\prime}+\beta\left(I_{p}^{m+1}(n ; \lambda, l) f(z)\right)^{\prime}\right]\right\}>\alpha,
$$

for $|z|<R$, where

$$
R=\left[\sqrt{1+\left(\frac{\beta \lambda}{l}\right)^{2}(p+n)^{2}}-\frac{\beta \lambda}{l}(p+n)\right]^{1 /(p+n)} .
$$

The result is the best possible.

PROOF. If we let

$$
-z^{p+1}\left(I_{p}^{m}(n ; \lambda, l) f(z)\right)^{\prime}=\alpha+(p-\alpha) \varphi(z),
$$

then $\varphi$ has the form (2.1), and is analytic with positive real part in $U$. Using the identity (1.2) in (3.5), and differentiating the resulting equation with respect to $z$,

$$
\begin{aligned}
& -\frac{z^{p+1}\left[(1-\beta)\left(I_{p}^{m}(n ; \lambda, l) f(z)\right)^{\prime}+\beta\left(I_{p}^{m+1}(n ; \lambda, l) f(z)\right)^{\prime}\right]+\alpha}{p-\alpha} \\
& =\varphi(z)+\frac{\beta \lambda}{l} z \varphi^{\prime}(z) .
\end{aligned}
$$

Applying in (3.6) the estimate (see [8])

$$
\frac{\left|z \varphi^{\prime}(z)\right|}{\operatorname{Re} \varphi(z)} \leq \frac{2(p+n) r^{p+n}}{1-r^{2(p+n)}}, \quad|z|=r<1,
$$

we get

$$
\begin{aligned}
\operatorname{Re}\{ & \left.-\frac{z^{p+1}\left[(1-\beta)\left(I_{p}^{m}(n ; \lambda, l) f(z)\right)^{\prime}+\beta\left(I_{p}^{m+1}(n ; \lambda, l) f(z)^{\prime}\right]+\alpha\right.}{p-\alpha}\right\} \\
& \geq\left[1-\frac{2 \beta \lambda}{l} \frac{(p+n) r^{p+n}}{1-r^{2(p+n)}}\right] \operatorname{Re} \varphi(z),
\end{aligned}
$$


and it is easy to see that the right-hand side of (3.7) is positive, provided that $r<R$, where $R$ is given by (3.4).

In order to show that the bound $R$ is the best possible, we consider the function $f \in \sum_{p, n}$ defined by

$$
-z^{p+1}\left(I_{p}^{m}(n ; \lambda, l) f(z)\right)^{\prime}=\alpha+(p-\alpha) \frac{1+z^{p+n}}{1-z^{p+n}} .
$$

Then

$$
\begin{gathered}
-\frac{z^{p+1}\left[(1-\beta)\left(I_{p}^{m}(n ; \lambda, l) f(z)\right)^{\prime}+\beta\left(I_{p}^{m+1}(n ; \lambda, l) f(z)\right)^{\prime}\right]+\alpha}{p-\alpha} \\
=\frac{1-z^{2(p+n)}+\frac{2 \beta \lambda}{l}(p+n) z^{p+n}}{\left(1-z^{p+n}\right)^{2}}=0,
\end{gathered}
$$

for $z=R \exp (i \pi /(p+n))$, which completes the proof of the theorem.

REMARK 3.7. Putting $\lambda=l=1$ in Theorem 3.6, we obtain the result of Srivastava and Patel [16, Theorem 2].

For $\beta=1$, Theorem 3.6 reduces to the following result.

Corollary 3.8. If the function $f \in \sum_{p, n}^{m}(\lambda, l ; \alpha), \quad 0 \leq \alpha<p$, then $f \in \sum_{p, n}^{m+1}(\lambda, l ; \alpha)$ for $|z|<\widetilde{R}$, where

$$
\widetilde{R}=\left[\sqrt{1+\left(\frac{\lambda}{l}\right)^{2}(p+n)^{2}}-\frac{\lambda}{l}(p+n)\right]^{1 /(p+n)} .
$$

The result is the best possible.

THEOREM 3.9. Let $f \in \sum_{p, n}^{m}(\lambda, l ; A, B)$, and let

$$
F_{p, c}(f)(z)=\frac{c}{z^{c+p}} \int_{0}^{z} t^{c+p-1} f(t) d t, \quad c>0 .
$$

Then

$$
-\frac{z^{p+1}\left(I_{p}^{m}(n ; \lambda, l) F_{p, c}(f)(z)\right)^{\prime}}{p} \prec \Theta(z) \prec \frac{1+A z}{1+B z},
$$

where $\Theta$ is defined by

$$
\Theta(z)= \begin{cases}\frac{A}{B}+\left(1-\frac{A}{B}\right)(1+B z)^{-1} F_{1}\left(1,1, \frac{c}{p+n}+1 ; \frac{B z}{1+B z}\right), & B \neq 0 \\ 1+\frac{A c}{c+p+n} z, & B=0\end{cases}
$$

and it is the best dominant of (3.9). 
Furthermore,

$$
\operatorname{Re}\left[-\frac{z^{p+1}\left(I_{p}^{m}(n ; \lambda, l) F_{p, c}(f)(z)\right)^{\prime}}{p}\right]>k, \quad z \in U,
$$

where $k=\Theta(-1)$, and this inequality is the best possible.

PROOF. Setting

$$
\varphi(z)=-\frac{z^{p+1}\left(I_{p}^{m}(n ; \lambda, l) F_{p, c}(f)(z)\right)^{\prime}}{p},
$$

then $\varphi$ has the form (2.1), and is analytic in $U$. Using in (3.10) the operator identity

$$
z\left(I_{p}^{m}(n ; \lambda, l) F_{p, c}(f)(z)\right)^{\prime}=c I_{p}^{m}(n ; \lambda, l) f(z)-(c+p)\left(I_{p}^{m}(n ; \lambda, l) F_{p, c}(f)(z)\right),
$$

and differentiating the resulting equation with respect to $z$, we find that

$$
-\frac{z^{p+1}\left(I_{p}^{m}(n ; \lambda, l) f(z)\right)^{\prime}}{p}=\varphi(z)+\frac{z \varphi^{\prime}(z)}{c} \prec \frac{1+A z}{1+B z} .
$$

Now, the remaining part of the proof follows by employing the same techniques that we used in the proof of Theorem 3.1.

REMARK 3.10. (1) Setting $n=0$ and $l=\lambda=1$ in Theorem 3.9, we obtain the following result which improves the corresponding work of Liu and Srivastava [7, Theorem 2]. If $c>0$ and $f \in R_{m, p}(A, B)$, then

$$
F_{p, c}\left(R_{m, p}(A, B)\right) \subset R_{m, p}(1-2 \zeta,-1) \subset R_{m, p}(A, B),
$$

where

$$
\zeta= \begin{cases}\frac{A}{B}+\left(1-\frac{A}{B}\right)(1-B)^{-1}{ }_{2} F_{1}\left(1,1, \frac{c}{p}+1 ; \frac{B}{B-1}\right), & B \neq 0, \\ 1-\frac{A c}{c+p}, & B=0 .\end{cases}
$$

The result is the best possible.

(2) Observing that

$$
z^{p+1}\left(I_{p}^{m}(n ; \lambda, l) F_{p, c}(f)(z)\right)^{\prime}=\frac{c}{z^{c}} \int_{0}^{z} t^{c+p}\left(I_{p}^{m}(n ; \lambda, l) f(t)\right)^{\prime} d t,
$$

whenever $f \in \sum_{p, n}$ and $c>0$, the above remark can be restated as follows. If $c>0$ and $f \in R_{m, p}(A, B)$, then

$$
\operatorname{Re}\left[-\frac{c}{p z^{c}} \int_{0}^{z} t^{c+p}\left(I_{p}^{m}(n ; \lambda, l) f(t)\right)^{\prime} d t\right]>\zeta, \quad z \in U,
$$

where $\zeta$ is given by (3.11). 
According to (3.12), and taking in the above theorem $A=1-2 \alpha / p, B=-1$, and $m=0$, we obtain the following special case.

COROLlary 3.11. If $c>0$ and if $f \in \sum_{p, n}$ satisfies the inequality

$$
\operatorname{Re}\left[-z^{p+1} f^{\prime}(z)\right]>\alpha, \quad z \in U(0 \leq \alpha<p),
$$

then

$$
\begin{aligned}
\operatorname{Re}[ & \left.-\frac{c}{z^{c}} \int_{0}^{z} t^{c+p} f^{\prime}(t) d t\right] \\
& >\alpha+(p-\alpha)\left[{ }_{2} F_{1}\left(1,1, \frac{c}{p+n}+1 ; \frac{1}{2}\right)-1\right], \quad z \in U,
\end{aligned}
$$

and the inequality is the best possible.

Using the technique of Srivastava and Patel [16, Theorem 4], we can prove the next theorem.

THEOREM 3.12. Let the function $f \in \sum_{p, n}$, and suppose that $g \in \sum_{p, n}$ satisfies the inequality

$$
\operatorname{Re}\left[z^{p} I_{p}^{m}(n ; \lambda, l) g(z)\right]>0, \quad z \in U
$$

If

$$
\left|\frac{I_{p}^{m}(n ; \lambda, l) f(z)}{I_{p}^{m}(n ; \lambda, l) g(z)}-1\right|<1, \quad z \in U\left(m \in \mathbb{N}_{0}, l, \lambda>0\right),
$$

then

$$
\operatorname{Re}\left[-\frac{z\left(I_{p}^{m}(n ; \lambda, l) f(z)\right)^{\prime}}{I_{p}^{m}(n ; \lambda, l) f(z)}\right]>0
$$

for $|z|<R_{0}$, where

$$
R_{0}=\frac{\sqrt{g(p+n)^{2}+4 p(2 p+n)}-3(p+n)}{2(2 p+n)} .
$$

PROOF. Letting

$$
w(z)=\frac{I_{p}^{m}(n ; \lambda, l) f(z)}{I_{p}^{m}(n ; \lambda, l) g(z)}-1=k_{p+n} z^{p+n}+k_{p+n+1} z^{p+n+1}+\cdots,
$$

then $w$ is analytic in $U$, with $w(0)=0,|w(z)|<1$ for all $z \in U$, and $w(z)=$ $k_{p+m} z^{p+m}+k_{p+m+1} z^{p+m+1}+\cdots$. Defining the function $\psi$ by

$$
\psi(z)= \begin{cases}\frac{w(z)}{z^{p+m}}, & z \in \dot{U}, \\ \frac{w^{(p+m)}(0)}{(p+m) !}, & z=0,\end{cases}
$$


then $\psi$ is analytic in $\dot{U}$ and continuous in $U$, hence it is analytic in the whole unit disc $U$. If $r \in(0,1)$ is an arbitrary number, since $|w(z)|<1$ for all $z \in U$, we deduce that

$$
|\psi(z)| \leq \max _{|z|=r}\left|\frac{w(z)}{z^{p+m}}\right| \leq \max _{|z|=r} \frac{|w(z)|}{|z|^{p+m}}<\frac{1}{r^{p+m}}, \quad|z| \leq r<1 .
$$

By letting $r \rightarrow 1^{-}$in the above inequality, we get $|\psi(z)|<1$ for all $z \in U$, that is, $w(z)=z^{p+n} \psi(z)$, where the function $\psi$ is analytic in $U$, and $|\psi(z)|<1, z \in U$.

Therefore, $(3.14)$ leads us to

$$
I_{p}^{m}(n ; \lambda, l) f(z)=I_{p}^{m}(n ; \lambda, l) g(z)\left(1+z^{p+n} \psi(z)\right), \quad z \in U,
$$

and differentiating logarithmically the above relation, we obtain

$$
\frac{z\left(I_{p}^{m}(n ; \lambda, l) f(z)\right)^{\prime}}{I_{p}^{m}(n ; \lambda, l) f(z)}=\frac{z\left(I_{p}^{m}(n ; \lambda, l) g(z)\right)^{\prime}}{I_{p}^{m}(n ; \lambda, l) g(z)}+\frac{z^{p+n}\left[(p+n) \psi(z)+z \psi^{\prime}(z)\right]}{1+z^{p+n} \psi(z)} .
$$

Setting $\varphi(z)=z^{p}\left(I_{p}^{m}(n ; \lambda, l) g(z)\right)$, we see that the function $\varphi$ has the form (2.1), is analytic in $U$ with $\operatorname{Re} \varphi(z)>0$, for all $z \in U$, and

$$
\frac{z\left(I_{p}^{m}(n ; \lambda, l) g(z)\right)^{\prime}}{I_{p}^{m}(n ; \lambda, l) g(z)}=\frac{z \varphi^{\prime}(z)}{\varphi(z)}-p .
$$

Hence, from (3.15) we find that

$$
\operatorname{Re}\left[-\frac{z\left(I_{p}^{m}(n ; \lambda, l) f(z)\right)^{\prime}}{I_{p}^{m}(n ; \lambda, l) f(z)}\right] \geq p-\left|\frac{z \varphi^{\prime}(z)}{\varphi(z)}\right|-\left|\frac{z^{p+n}\left[(p+n) \psi(z)+z \psi^{\prime}(z)\right]}{1+z^{p+n} \psi(z)}\right| .
$$

Now, by using in (3.16) the known estimates (see [8])

$$
\begin{gathered}
\left|\frac{\varphi^{\prime}(z)}{\varphi(z)}\right| \leq \frac{2(p+n) r^{p+n-1}}{1-r^{2(p+n)}}, \quad|z|=r<1, \\
\left|\frac{(p+n) \psi(z)+z \psi^{\prime}(z)}{1+z^{p+n} \psi(z)}\right| \leq \frac{p+n}{1-r^{(p+n)}}, \quad|z|=r<1,
\end{gathered}
$$

we conclude that

$$
\operatorname{Re}\left[-\frac{z\left(I_{p}^{m}(n ; \lambda, l) f(z)\right)^{\prime}}{I_{p}^{m}(n ; \lambda, l) f(z)}\right] \geq \frac{p-3(p+n) r^{p+n}-(2 p+n) r^{2(p+n)}}{1-r^{2(p+n)}},
$$

for $|z|=r<1$, which is positive provided that $r<R_{0}$, where $R_{0}$ is given by (3.13).

THEOREM 3.13. Let $-1 \leq B_{i}<A_{i} \leq 1, i=1,2$, and suppose that each of the functions $f_{i} \in \sum_{p}$ satisfies the subordination condition

$$
(1-\beta) z^{p} I_{p}^{m}(\lambda, l) f_{i}(z)+\beta z^{p} I_{p}^{m+1}(\lambda, l) f_{i}(z) \prec \frac{1+A_{i} z}{1+B_{i} z}, \quad i=1,2,
$$


where $I_{p}^{m}(\lambda, l) \equiv I_{p}^{m}(-p+1 ; \lambda, l)$. Then

$$
(1-\beta) z^{p} I_{p}^{m}(\lambda, l) G(z)+\beta z^{p} I_{p}^{m+1}(\lambda, l) G(z) \prec \frac{1+(1-2 \eta) z}{1-z},
$$

where

$$
G(z)=I_{p}^{m}(\lambda, p)\left(f_{1} * f_{2}\right)(z)
$$

and

$$
\eta=1-\frac{4\left(A_{1}-B_{1}\right)\left(A_{2}-B_{2}\right)}{\left(1-B_{1}\right)\left(1-B_{2}\right)}\left[1-{ }_{2} F_{1}\left(1,1, \frac{l}{\beta \lambda}+1 ; \frac{1}{2}\right)\right] .
$$

The result is the best possible when $B_{1}=B_{2}=-1$.

PROOF. Since each of the functions $f_{i} \in \sum_{p}, i=1,2$, satisfies condition (3.17), then by letting

$$
\varphi_{i}(z)=(1-\beta) z^{p} I_{p}^{m}(\lambda, l) f_{i}(z)+\beta z^{p} I_{p}^{m+1}(\lambda, l) f_{i}(z), \quad i=1,2,
$$

we have

$$
\varphi_{i} \in \mathcal{P}\left(\gamma_{i}\right) \quad \text { where } \gamma_{i}=\frac{1-A_{i}}{1-B_{i}}(i=1,2) .
$$

Using identity (1.2) in (3.18),

$$
I_{p}^{m}(\lambda, l) f_{i}(z)=\frac{l}{\beta \lambda} z^{-p-l / \beta \lambda} \int_{0}^{z} t^{(l / \beta \lambda)-1} \varphi_{i}(t) d t, \quad i=1,2,
$$

which, according to the definition of $G$, yields

$$
I_{p}^{m}(\lambda, l) G(z)=\frac{l}{\beta \lambda} z^{-p-l / \beta \lambda} \int_{0}^{z} t^{(l / \beta \lambda)-1} \varphi_{0}(t) d t,
$$

where

$$
\begin{aligned}
\varphi_{0}(z) & =(1-\beta) z^{p} I_{p}^{m}(\lambda, l) G(z)+\beta z^{p} I_{p}^{m+1}(\lambda, l) G(z) \\
& =\frac{l}{\beta \lambda} z^{-l / \beta \lambda} \int_{0}^{z} t^{(l / \beta \lambda)-1}\left(\varphi_{1} * \varphi_{2}\right)(t) d t .
\end{aligned}
$$

Since $\varphi_{i} \in \mathcal{P}\left(\gamma_{i}\right), i=1,2$, it follows from Lemma 2.4 that

$$
\varphi_{1} * \varphi_{2} \in \mathcal{P}\left(\gamma_{3}\right) \quad \text { where } \gamma_{3}=1-2\left(1-\gamma_{1}\right)\left(1-\gamma_{2}\right) .
$$

By using (3.20) and (3.19), from Lemmas 2.3 and 2.6, we get

$$
\begin{aligned}
\operatorname{Re} \varphi_{0}(z) & =\frac{l}{\beta \lambda} z^{-l / \beta \lambda} \int_{0}^{1} u^{(l / \beta \lambda)-1} \operatorname{Re}\left(\varphi_{1} * \varphi_{2}\right)(u z) d u \\
& \geq \frac{l}{\beta^{\lambda}} \int_{0}^{1} u^{\left(l / \beta^{\lambda}\right)-1}\left[2 \gamma_{3}-1+\frac{2\left(1-\gamma_{3}\right)}{1+u|z|}\right] d u \\
& >\frac{l}{\beta \lambda} \int_{0}^{1} u^{(l / \beta \lambda)-1}\left[2 \gamma_{3}-1+\frac{2\left(1-\gamma_{3}\right)}{1+u}\right] d u
\end{aligned}
$$




$$
\begin{aligned}
& =1-\frac{4\left(A_{1}-B_{1}\right)\left(A_{2}-B_{2}\right)}{\left(1-B_{1}\right)\left(1-B_{2}\right)}\left[1-\frac{l}{\beta \lambda} \int_{0}^{1} u^{(l / \beta \lambda)-1}(1+u)^{-1} d u\right] \\
& =1-\frac{4\left(A_{1}-B_{1}\right)\left(A_{2}-B_{2}\right)}{\left(1-B_{1}\right)\left(1-B_{2}\right)}\left[1-\frac{1}{2}{ }_{2} F_{1}\left(1,1, \frac{l}{\beta \lambda}+1 ; \frac{1}{2}\right)\right]=\eta, \quad z \in U .
\end{aligned}
$$

When $B_{1}=B_{2}=-1$, consider the functions $f_{i} \in \sum_{p}, i=1,2$, which satisfy assumptions (3.17) and are defined by

$$
I_{p}^{m}(\lambda, l) f_{i}(z)=\frac{l}{\beta \lambda} z^{-l / \beta \lambda} \int_{0}^{z} t^{(l / \beta \lambda)-1}\left(\frac{1+A_{i} t}{1-t}\right) d t, \quad i=1,2 .
$$

Thus, from (3.19) and Lemma 2.6, it follows that

$$
\begin{aligned}
\varphi_{0}(z)= & \frac{l}{\beta \lambda} \int_{0}^{1} u^{(l / \beta \lambda)-1}\left[1-\left(1+A_{1}\right)\left(1+A_{2}\right)+\frac{\left(1+A_{1}\right)\left(1+A_{2}\right)}{1-u z}\right] d u \\
= & 1-\left(1+A_{1}\right)\left(1+A_{2}\right)+\left(1+A_{1}\right)\left(1+A_{2}\right)(1-z)^{-1} \\
& \times{ }_{2} F_{1}\left(1,1, \frac{l}{\beta \lambda}+1 ; \frac{z}{z-1}\right) \\
\rightarrow & 1-\left(1+A_{1}\right)\left(1+A_{2}\right)+\frac{1}{2}\left(1+A_{1}\right)\left(1+A_{2}\right)_{2} F_{1}\left(1,1, \frac{l}{\beta \lambda}+1 ; \frac{1}{2}\right),
\end{aligned}
$$

as $z \rightarrow-1$, which completes the proof.

Taking $A_{i}=1-2 \alpha_{i}, B_{i}=-1(i=1,2), m=0$ and $l=\lambda=1$ in Theorem 3.13, we obtain the following result which refines the work of Yang [20, Theorem 4].

COROLlary 3.14. If the functions $f_{i} \in \sum_{p}, i=1$, 2, satisfy the inequality

$$
\operatorname{Re}\left\{(1+\beta p) z^{p} f_{i}(z)+\beta z^{p+1} f_{i}^{\prime}(z)\right\}>\alpha_{i}, \quad z \in U\left(0 \leq \alpha_{i}<1, i=1,2\right),
$$

then

$$
\operatorname{Re}\left\{(1+\beta p) z^{p}\left(f_{1} * f_{2}\right)(z)+\beta z^{p+1}\left(f_{1} * f_{2}\right)(z)\right\}>\eta_{0}, \quad z \in U,
$$

where

$$
\eta_{0}=1-4\left(1-\alpha_{1}\right)\left(1-\alpha_{2}\right)\left[1-\frac{1}{2}{ }_{2} F_{1}\left(1,1, \frac{1}{\beta}+1 ; \frac{1}{2}\right)\right] .
$$

The result is the best possible.

THEOREM 3.15. If the function $f \in \sum_{p, n}$ satisfies the subordination condition

$$
(1-\beta) z^{p} I_{p}^{m}(n ; \lambda, l) f(z)+\beta z^{p} I_{p}^{m+1}(n ; \lambda, l) f(z) \prec \frac{1+A z}{1+B z},
$$

then

$$
\operatorname{Re}\left[z^{p} I_{p}^{m}(n ; \lambda, l) f(z)\right]^{1 / q}>\rho^{1 / q}, \quad z \in U(q \in \mathbb{N}),
$$

where $\rho=Q(-1)$ is given as in Theorem 3.1. The result is the best possible. 
PROOF. Defining the function $\varphi$ by

$$
\varphi(z)=z^{p} I_{p}^{m}(n ; \lambda, l) f(z),
$$

we see that $\varphi$ has the form (2.1) and is analytic in $U$. Using identity (1.2) in (3.22), and differentiating the resulting equation with respect to $z$, we obtain

$$
(1-\beta) z^{p} I_{p}^{m}(n ; \lambda, l) f(z)+\beta z^{p} I_{p}^{m+1}(n ; \lambda, l) f(z)=\varphi(z)+\frac{\beta \lambda}{l} z \varphi^{\prime}(z) \prec \frac{1+A z}{1+B z} .
$$

Now, by following similar steps to the proof of Theorem 3.1, and using the elementary inequality

$$
\operatorname{Re} w^{1 / q} \geq(\operatorname{Re} w)^{1 / q}, \quad \operatorname{Re} w>0, q \in \mathbb{N}
$$

we obtain the result asserted by Theorem 3.15.

From Corollary 3.14 and Theorem 3.15, for the special case $n=-p+1, m=0$, $A=1-2 \eta_{0}, B=-1$ and $q=1$, we deduce the next result.

Corollary 3.16. Let the functions $f_{i} \in \sum_{p}(i=1,2)$, satisfy inequality (3.21). Then

$$
\operatorname{Re}\left[z^{p}\left(f_{1} * f_{2}\right)(z)\right]>\eta_{0}+\left(1-\eta_{0}\right)\left[{ }_{2} F_{1}\left(1,1, \frac{1}{\beta}+1 ; \frac{1}{2}\right)-1\right], \quad z \in U,
$$

where $\eta_{0}$ is given as in Corollary 3.14. The result is the best possible.

THEOREM 3.17. If the function $g \in \sum_{p, n}$ satisfies the inequality

$$
\operatorname{Re}\left[z^{p} g(z)\right]>\frac{1}{2}, \quad z \in U,
$$

then, for any function $f \in \sum_{p, n}^{m}(\lambda, l, A ; B)$, we have

$$
f * g \in \sum_{p, n}^{m}(\lambda, l ; A, B) .
$$

PROOF. It is easy to check that

$$
-\frac{z^{p+1}\left(I_{p}^{m}(n ; \lambda, l)(f * g)(z)\right)^{\prime}}{p}=\left[-\frac{z^{p+1}\left(I_{p}^{m}(n ; \lambda, l) f(z)\right)^{\prime}}{p}\right] *\left[z^{p} g(z)\right] .
$$

According to this relation, by applying Lemma 2.5 for the functions

$$
F(z)=-\frac{z^{p+1}\left(I_{p}^{m}(n ; \lambda, l) f(z)\right)^{\prime}}{p}
$$

and $\Phi(z)=z^{p} g(z)$, and using the fact that the function $h(z)=(1+A z) /(1+B z)$ is convex (univalent) in $U$, we deduce the conclusion of the theorem. 


\section{References}

[1] M. K. Aouf and H. M. Hossen, 'New criteria for meromorphic $p$-valent starlike functions', Tsukuba J. Math. 17 (1993), 481-486.

[2] N. E. Cho, O. S. Kwon and H. M Srivastava, 'Inclusion relationships and argument properties for certain subclasses of multivalent functions associated with a family of linear operators', J. Math. Anal. Appl. 242 (2004), 470-480.

[3] N. E. Cho, O. S. Kwon and H. M Srivastava, 'Inclusion and argument properties for certain subclasses of meromorphic functions associated with a family of multiplier transformations', J. Math. Anal. Appl. 300 (2004), 505-520.

[4] N. E. Cho, O. S. Kwon and H. M. Srivastava, 'Inclusion relationships for certain subclasses of meromorphic functions associated with a family of multiplier transformations', Integral Transforms Spec. Funct. 16(18) (2005), 647-659.

[5] D. J. Hallenbeck and St. Ruscheweyh, 'Subordinations by convex functions', Proc. Amer. Math. Soc. 52 (1975), 191-195.

[6] J.-L. Liu and H. M. Srivastava, 'A linear operator and associated families of multivalent functions', J. Math. Anal. Appl. 259 (2001), 566-581.

[7] J.-L. Liu and H. M. Srivastava, 'Subclasses of meromorphically multivalent functions associated with a certain linear operator', Math. Comput. Modelling 39 (2004), 35-44.

[8] T. H. MacGregor, 'Radius of univalence of certain analytic functions', Proc. Amer. Math. Soc. 14 (1963), 514-520.

[9] S. S. Miller and P. T. Mocanu, 'Second order differential inequalities in the complex plane', J. Math. Anal. Appl. 65 (1978), 289-305.

[10] S. S. Miller and P. T. Mocanu, Differential Subordinations. Theory and Applications, Series on Monographs and Textbooks in Pure and Applied Mathematics, 225 (Marcel Dekker Inc., New York, 2000).

[11] M. L. Mogra, 'Meromorphic multivalent functions with positive coefficients. I', Math. Japon. 35(1) (1990), 1-11.

[12] M. L. Mogra, 'Meromorphic multivalent functions with positive coefficients. II', Math. Japon. 35(6) (1990), 1089-1098.

[13] M. Pap, 'On certain subclasses of meromorphic $m$-valent close-to-convex functions', Pure Math. Appl. 9 (1998), 155-163.

[14] D. Ž. Pashkouleva, 'The starlikeness and spiral-convexity of certain subclasses of analytic functions', in: Current Topics in Analytic Function Theory (eds. H. M. Srivastava and S. Owa) (World Scientific Publishing Company, Singapore, 1992).

[15] R. Singh and S. Singh, 'Convolution properties of a class of starlike functions', Proc. Amer. Math. Soc. 106 (1989), 145-152.

[16] H. M. Srivastava and J. Patel, 'Applications of differential subordination to certain classes of meromorphically multivalent functions', J. Inequal. Pure Appl. Math. 6(3) (2005), 15.

[17] J. Stankiewicz and Z. Stankiewicz, 'Some applications of the Hadamard convolution in the theory of functions', Ann. Univ. Mariae Curie-Sklodowska Sect. A 40 (1986), 251-265.

[18] B. A. Uralegaddi and C. Somanatha, 'New criteria for meromorphic starlike univalent functions', Bull. Aust. Math. Soc. 43 (1991), 137-140.

[19] E. T. Whittaker and G. N. Watson, A Course of Modern Analysis: An Introduction to the General Theory of Infinite Processes and of Analytic Functions: With an Account of the Principal Transcendental Functions, 4th edn (Cambridge University Press, Cambridge, 1927).

[20] D.-G. Yang, 'Certain convolution operators for meromorphic functions', South East Asian Bull. Math. 25 (2001), 175-186.

\section{R. M. EL-ASHWAH, Department of Mathematics, Faculty of Science, Mansoura University, Mansoura 35516, Egypt \\ e-mail: r_elashwah@yahoo.com}


M. K. AOUF, Department of Mathematics, Faculty of Science, Mansoura University, Mansoura 35516, Egypt

e-mail: mkaouf127@yahoo.com

T. BULBOACĂ, Faculty of Mathematics and Computer Science,

Babeş-Bolyai University, 400084 Cluj-Napoca, Romania

e-mail: bulboaca@math.ubbcluj.ro 